\title{
Results and Prospects of Experimental Validation of the Use of Electromagnetic Waves at Terahertz Frequencies of Cellular Metabolites in Clinical Practice
}

\author{
V. F. Kirichuk \\ Department of Normal Physiology, Saratov State Medical University, Saratov, Russia \\ Email: normalf@yandex.ru
}

Received 25 September 2013; accepted 25 May 2015; published 28 May 2015

Copyright (C) 2015 by author and Scientific Research Publishing Inc.

This work is licensed under the Creative Commons Attribution International License (CC BY). http://creativecommons.org/licenses/by/4.0/

(c) (i) Open Access

\begin{abstract}
The paper presents the results of experimental studies of the effect of irradiation with electromagnetic waves terahertz frequencies of molecular spectrum of emission and absorption of nitric oxide and atmospheric oxygen on animals in a state of acute and chronic immobilization stress, a condition regarded as a model of violations in different parts of the vascular system including microcirculation specific for different parts of the vascular system, including the microcirculation characteristic of various diseases of the cardiovascular system.
\end{abstract}

Keywords

Terahertz Waves, Microcirculation, Acute Stress

\section{Introduction}

Electromagnetic radiation in the terahertz frequency range is now increasingly beginning to be applied mainly as a correction factor of microcircular violations analysis of the literature, and clinical observations suggest that MM-wave is effective for many chronic diseases as monotherapy in combination with other physical methods and medication, strengthening and consolidating the therapeutic effect [1]-[6].

Terahertz frequency range (TGCH) lies on the border between electronics and photonics from 100 GGts to 10 TGts $(1 \mathrm{THz}=10 \mathrm{Z} \mathrm{GHz})$ or in wavelengths from $3 \mathrm{~mm}$ to $30 \mathrm{~mm}$. It is found that the considered wavelength range is used by living organisms for communication and control, and that the organisms themselves emit vibrations millimeter waves excited by the body when exposed to TGCH exposure, and to some extent mimic the 
signals of internal communication and control (data communication) of biological objects. The result is reduced by the normal range and power of the radiation characteristic of the organism, thus representing that a range of frequencies do not qualitatively change the body, but can be adjusted to normalize its physiological state within specific to the biological species.

It is believed that the reactivity of molecules excited terahertz quantum will also include what TGCH radiation easily penetrates through clothing and skin to the muscle man.

On the other hand, the terahertz frequency range increasingly attracts attention, because of this frequency range centered emission and absorption spectra critical cellular metabolites- $-\mathrm{NO}_{2}, \mathrm{CO}_{2}, \mathrm{CO}, \mathrm{OH}$, etc. [2] .

Naturally, the greatest interest is the molecular spectrum of electromagnetic waves of radiation and absorption of nitric oxide. It is one of the most important biological mediators involved in many physiological and pathophysiological processes, acting as a second messenger in most cells. In particular, nitric oxide is involved in the implementation of many important physiological functions, such as vasodilation, bronchodilation, neurotransmission, platelet aggregation, immune system reactions, the regulation of smooth muscle tone, and others [6].

Research and development of methods to control the secretion of maintaining the level of physiological reactivity and concentration of endogenous nitric oxide in the cells, organs and the whole body are scientific and of practical interest for theoretical and clinical medicine. In this regard, currently, intensive search for methods is constantly conducted to create pharmaceutical preparations regulating the secretion and physiological activity of nitric oxide molecules in cells, organs, and body as a whole. However, pharmacological regulation altered physiological functions in the body may be accompanied by the appearance of some persistent undesirable side effects, and in some cases by severe allergic reactions, which limit the breadth of application of medicaments.

This dictates the need to find non-invasive physical controllers formation and secretion of endogenous nitric oxide on the basis of natural physiological processes. The use of low-intensity terahertz radiation at frequencies of molecular spectrum of emission and absorption of nitric oxide and atmospheric oxygen is promising in this respect.

According to the contract No. 005/037/002 on September 25, 2001, Saratov State University and the Ministry of Health and Social Development of the Russian Federation at the Department of Physiology named by I. A. Chuevsky within the industry research program No. 9 "etiology and pathogenesis, diagnosis and treatment of diseases of the blood" are working on "studies on the effect of complex biological systems of electromagnetic waves at frequencies of molecular emission and absorption spectra of substances involved in metabolic processes". Agreements were signed on the joint scientific and technical activities between Saratov State Medical University and JSC "Central Research Institute of Measuring Equipment" (Saratov) - contract No. 67 on January 31, 2000, contract No. 73 on April 16, 2002 , contract No. 14 on March 28, 2006, as well as a research center for biophotonics Institute of Biomedical Engineering and Technology health and the Institute of Advanced technology (Shenzhen), Chinese Academy of Sciences - the agreement on March 2, 2010. In addition, studies were carried out under the program of Medical Sciences' scientific medical research Volga region for 2008-2010 on study of the behavioral responses, and the nature of changes in blood flow in the great vessels of blood rheology, microcircular and coagulation mechanisms of homeostasis in biological objects in a state of acute and chronic immobilization stress radiopulse under the influence of radiation of high power and different terahertz frequencies (135 - $250 \mathrm{GHz})$.

\section{Material and Methods}

The studies were conducted on 2175 adult white rats of both sexes weighing $150-200$ grams in conditions of acute and chronic stress. Used advanced studies of vascular and endovascular mechanisms of microcirculation. Exposure of rats was terahertz electromagnetic waves at frequencies of molecular emission spectrum absorbtion nitric oxide (150.176 - $150.664 \mathrm{GHz})$ and atmospheric oxygen (129 GHz) Apparatus of "Orbit".

\section{Results and Discussion}

Based on studies conducted by staff of the Department of Physiology of the normal, I. A. Chuevsky experimentally uses clinical practice, the electromagnetic waves at different frequencies of molecular spectrum of emission and absorption of nitric oxide and oxygen in the intravascular and vascular units microcirculation, the functional activity of the endocrine glands, states lipid peroxidation and antioxidant activity of blood, the behavioral responses of animals, and antistress activity of the body. 
Investigations were carried out in two directions, the in vitro and in vivo in two models of breach of these indicators' homeostatic responses of animals (white rats of both sexes), and acute and long-term stress, which were the typical pathological process in various diseases of the cardio-vascular system, malignant diseases of various inflammatory processes.

In vitro studies on platelet-rich plasma in patients with unstable angina showed a normalizing effect of electromagnetic waves of extremely high and terahertz frequency band of the molecular spectrum of emission and absorption of nitric oxide on platelet hemostasis, whose intravascular component of the microcirculation in the comparative evaluation of different modes of electromagnetic millimeter waves for the first time identified that the view EHF radiation was the most efficient for the normalization of activity and platelet aggregation in an in vitro (amplitude nomodulirovannoe effect on the frequency of $42.2 \mathrm{GHz}$ ). First, it studied the effects of platelet-THF-radiation at frequencies of molecular spectrum of emission and absorption of nitric oxide 150.176 $150.664 \mathrm{GHz}$ mode. TGCH effects on specified frequencies of molecular spectrum of emission and absorption of nitric oxide caused the most pronounced normalization of functional activity of platelets in patients with unstable angina (15-minute exposure, and continuous signal generation). A comparative analysis of platelet effects of EHF-exposure and impacts on the frequency spectrum of the molecular absorption and emission of nitrogen oxide shows a more pronounced effect of the latter on the functional activity (activation and aggregation) of platelets in patients with unstable angina. For the first time, it shows the possibility of information impact of intact platelets of healthy subjects of patients with unstable angina platelets subjected TCHG-radiation at frequencies of molecular spectrum of emission and absorption of nitric oxide $150.176-150.664 \mathrm{GHz}$.

The effect of electromagnetic radiation in the frequency range terahertz molecular spectrum of emission and absorption of nitric oxide $240 \mathrm{GHz}$ on platelet aggregation, blood rheology, coagulation potential and fibrinolytic activity of the blood in patients with unstable angina in the conditions shows that irradiation of terahertz waves at a frequency of molecular spectrum of emission and absorption nitric oxide $240 \mathrm{GHz}$ in the in vitro leads to the normalization of indicators characterizing platelet aggregation, blood rheology, coagulation and fibrinolytic potential of the activity of the blood in patients with unstable angina.

The dependence of the efficiency of the exposure effects of electromagnetic radiation in the terahertz frequency range of molecular absorption and emission of nitrogen oxide $240 \mathrm{GHz}$ under in vitro conditions for the changed parameters of platelet function, the rheological properties of blood (blood viscosity, aggregation and deformability of red blood cells), and procoagulant, anticoagulant and fibrinolytic activity of the blood in the natural electromagnetic field of patients with unstable angina most effective in restoring altered blood parameters is a 15-minute exposure mode.

There is a dependence of the efficiency of the exposure effects of electromagnetic radiation in the terahertz frequency range of molecular nitrogen oxide in the in vitro conditions for the changed parameters of platelet function, rheology coagulation potential and fibrinolytic activity of the blood found in crossed magnetic fields and electrolytic, patients with unstable angina. The most effective in restoring altered blood parameters are $15-$ and 30-minute exposure modes.

For correction of impaired platelet function ballroom unstable angina in vitro used mode terahertz radiation waves at a frequency of $400 \mathrm{GHz}$ nitric oxide. The resulting positive result indicates the need for further study of the use of this mode of exposure to the changed parameters of the microcirculation in patients with cardiology. The rats are able to oxidative stress simulating similar changes in endothelial dysfunction in patients with unstable angina were increased platelet aggregation, blood rheology change - increased viscosity, erythrocyte aggregation and deformability blocked, a marked change in the hypercoagulable hemostasis and depression fibrinolytic activity of blood.

Found that the effect of the normalizing effect of $\mathrm{THz}$ radiation on the frequency spectrum of the molecular absorption and emission of nitrogen oxide $240 \mathrm{GHz}$ on the changed parameters of platelet aggregation, rheological properties of the coagulation and fibrinolytic potential of blood is based on the activation of endothelial NO synthase, responsible for the physiological synthesis of nitric oxide.

Thus, the experiment proved the prospect of using terahertz radiation at a frequency range of molecular absorption and emission of nitrogen oxide $240 \mathrm{GHz}$ in clinical practice for the normalization of the changed parameters of platelet aggregation, rheological properties of the coagulation and fibrinolytic potential of blood in patients with cardiology. There is a possibility of further study of terahertz radiation at a frequency range of molecular absorption and emission of nitrogen oxide $400 \mathrm{GHz}$ to justify its use in clinical practice. The influence of electromagnetic radiation TGCH -band frequency spectrum of the molecular emission and absorption of atmos- 
pheric oxygen $129 \mathrm{GHz}$ on the rheological properties of blood patchy, with 15- and 30-minute exposure is set lower the viscosity of whole blood, and at 60-minute exposure of its increase in the groups of patients with stable angina and almost healthy individuals. In this case, two modes of exposure cause opposite changes aggregation activity of the deformability of red blood cells and the ability of their membranes, and at 60-minute exposure - increasing the ability of red blood cell aggregation and a slight increase in ductility of red blood cell membranes.

The influence of electromagnetic radiation terahertz frequency MSIP nitric oxide $150.176-150.664 \mathrm{GHz}$ on the rheological properties of blood varies depending on exposure and radiation exposure resulted in an increase, as a rule, a more pronounced increase in the viscosity of whole blood of patients with stable angina and healthy individuals. A similar pattern was revealed with an increase in the radiation power. For all modes, exposure frequency EMR MSIP nitric oxide changes in functional parameters of red blood cells were found in different directions increase the ability of red blood cell aggregation and deformability decrease in membrane.

The influence of electromagnetic radiation frequencies $42.19 \mathrm{GHz}$ and $53 \mathrm{GHz}$ to 54 rheological parameters in patients with stable angina and healthy individuals is to increase the viscosity of whole blood by increasing the ability of the aggregation of red blood cells in patients with stable angina and in healthy individuals.

There is a significant dependence of the effect of EMI MMD blood cell peripheral blood at baseline Maximum severity of the rheological parameters of whole blood irradiation EMI MMD is determined by an optimal ratio of blood red cells, white cells and platelets.

Comparative analysis of the impact of EMR on the MMD "classical" frequencies (42.19 - 53.54 GHz) frequencies and MSIP nitric oxide and atmospheric oxygen showed that the severity of the effect of these frequencies EHF EMR on the rheological properties of blood are increased in the direction of 42, 19, 129, 53, 54, $150.176-150.664 \mathrm{GHz}$.

The severity of changes in the shape size, aggregating capacity of red blood cells, the plasticity of their membranes and cytometric changes due to the growth of the power supply and an increase in the exposure of EMR MMD all studied frequencies and for all modes of exposure. Effect of EMP MMD different frequencies on the viscosity of whole blood depends on the extent of atherosclerosis in the coronary arteries and the presence of hypertension increase with the number of coronary arteries, involved in the atherosclerotic process, increase efficiency index for the viscosity of whole blood erythrocyte aggregation and deformability of the membranes, the presence of hypertension patient leads to significantly higher efficiency index for the viscosity of whole blood, red blood cell aggregation and deformability of the ability of their membranes.

Identified "Information Impact" TGCH-irradiated whole blood in patients with stable angina and healthy individuals. Red blood cells are involved in the process of implementing one introduced EMI MMD "information signal" during irradiation frequency EMR 42, 19, 53, 54 and $150.176-150.664 \mathrm{GHz}$.

The effectiveness of the influence of electromagnetic radiation THF-MSIP frequency range $240 \mathrm{GHz}$ of nitric oxide in the in vitro disturbed viscous properties of whole blood aggregation ability and deformability of red blood cells in patients with unstable angina who are in the natural electromagnetic field depends on the duration of exposure. 15-minute exposure mode is most effective in restoring the disturbed viscosity properties of blood and red blood cell aggregation and deformability blocked - in 15- and 30-minute exposure.

Normalizing effect of terahertz radiation at a frequency of MSIP nitric oxide $240 \mathrm{GHz}$ in vitro on Ip broken viscous properties of whole blood erythrocyte aggregation and deformability in patients with unstable angina who are in crossed electric and magnet fields, depending on the time of exposure: the restoration of increased viscosity of whole blood is expressed in 15-minute exposure and impaired red blood cell deformability and aggregation - at 15- and 30-minute exposure. The specified mode of exposure is the most effective when compared with the influence of EMR THF $240 \mathrm{GHz}$ at the blood that is in the natural electromagnetic field, as it causes the improvement of the rheological properties of the majority of patients as well.

Normalizing effect on the frequency EMR THF MSIP nitric oxide $240 \mathrm{GHz}$ under in vitro conditions on the broken properties of whole blood viscosity, erythrocyte aggregation and deformability in patients with unstable angina is most marked and maintained for 30 minutes after irradiation. Irradiation at the frequency of MSIP 240 $\mathrm{GHz}$ nitric oxide nitric oxide donator Isoketum leads to a more severe degree of recovery altered blood viscosity and deformability of red blood cells in patients with unstable angina compared with non-irradiated Isoketum.

First studied the effects of electromagnetic radiation at frequencies terahertz range of molecular spectrum of nitric oxide 150, 176 - 150, and $664 \mathrm{GHz}$ on the functional activity of platelets in conditions in vivo.

The dependence of the efficiency effects of electromagnetic radiation in the terahertz frequency range of mo- 
lecular nitrogen oxide 150, 176 - 150, and $664 \mathrm{GHz}$ impaired functional activity of platelets in white male rats in a state of acute immobilization stress on the exposure mode and exposure. Thus, with continuous irradiation of animals in a state of acute immobilization stress, the greatest efficacy of the 30-minute exposure fractional THF-exposure of animals in a state of acute immobilization stress more effectively restores the basic platelet aggregation: 5- and 15-minute exposure more effective than the corresponding duration of continuous irradiation.

First studied the preventive effects of electromagnetic radiation at terahertz frequency range of molecular nitrogen oxide 150, 176 - 150, and $664 \mathrm{GHz}$ stressful violation of platelet function in male rats.

Found that prior immobilization TGCH exposure can prevent the development of stress disorders platelet function. It was found that 5- and 15-minute-exposure modes TGCH to immobilize have a more pronounced effect on platelet aggregation stressful violations compared with the same time of exposure modes on the background of established acute stress. The effectiveness of the 30-minute regime is the same for both methods of application of radiation, and that in both cases there is a complete recovery performance aggregation ability of platelets. First, to show that acute and chronic immobilization stress in male rats and female rats that are in one of the two phases of the estrous cycle or Dioestrus oestus observed violations of the coagulation and fibrinolytic properties of blood. White female rats respond to acute immobilization stress as a function of the estrous cycle in the phase Dioestrus, they are less sensitive to stress factors than in the phase Oestus.

The impact TGCH irradiation at frequencies MSIP nitric oxide 150, 176 - 150, and $664 \mathrm{GHz}$ continuously for 30 minutes in intact animals does not cause changes in the coagulant and fibrinolytic activity of the blood of male rats.

TGCH-radiation at frequencies MSIP nitric oxide 176 - 150.664, and $150 \mathrm{GHz}$ partially or completely normalizes disturbances in coagulation and fibrinolytic activity of blood uderstressed white male rats, depending on the time and mode of exposure.

For the first time found that when animals are irradiated continuously and with acute stress immobilization is most effective mode of 30-minute exposure. Prior to the action of irradiation of a stressor in the continuous mode prevents the development of irregularities in the different phases of blood coagulation and fibrinolysis, with 15-minute irradiation. The fractional mode of exposure of animals with acute immobilization stress is a full restoration of the studied parameters of coagulation hemostasis and fibrinolysis during the 15-minute TGC-influence at frequencies MSIP NO 150.176 - $150.664 \mathrm{GHz}$. Daily pre-exposure TGCH continuously for $30 \mathrm{mi}-$ nutes of male rats in a state of chronic immobilization stress, completely prevents disturbances in the blood coagulation system in fibrinolysis.

Comparative analysis of the effect of continuous and fractional modes of terahertz radiation at frequencies MSIP nitric oxide 176 - 150.664, and $150 \mathrm{GHz}$ performance coagulative hemostasis and fibrinolysis white male rats in a state of acute immobilization stress, showed that at the 15-minute TGCH-effects in the fractional mode showed complete normalization of disturbed blood coagulation parameters and blood fibrinolytic activity. Continuous exposure mode in the same time repartition showed only partial recovery of indicators of coagulation hemostasis and fibrinolysis.

Effect of pre-and post terahertz radiation at frequencies MSIP nitric oxide $150.176-150.664 \mathrm{GHz}$ performance coagulative and fibrinolytic activity of the blood of white male rats in a state of acute immobilization stress is ambiguous. The first shows that the pre-continuous irradiation uderstressed animals within 15 minutes completely prevents the development of disturbances in coagulation hemostasis and fibrinolysis. Continuous exposure mode on the background of established immobilization stress in the same time limit has only a partial recovery of blood coagulation activity indicators and fibrinolytic potential. First discovered features of the effect EMR THF -band frequencies on MSIP NO $150.176-150.664 \mathrm{GHz}$ on coagulation and hemostasis fibrinolid white female rats that are in one of the two phases of the estrous cycle Dioesrtus and Oestrus in acute immobilization stress as a function of the irradiation rats females continuously TGCH-waves at frequencies MSIP 176 $150.664 \mathrm{NO} 1 \mathrm{GHz}$ for 15 minutes to an action stressor agent completely prevented the development of disorders of blood coagulation potential and performance of fibrinolysis regardless of the phase of the estrous cycle. Fractional TGCH-radiation at frequencies MSIP NO $150.176-150.664 \mathrm{GHz}$ in the same exposure by an acute immobilization stress has only a partial restoration of indicators of coagulation hemostasis and fibrinolysis in female rats in phases Dioesrtus and Oestrus cycle.

It is shown that the most effective in the restoration of disturbed coagulation and fibrinolytic properties of blood in female rats in Dioesrtus phases of the estrous cycle and Oestrus is a preventive mode TGCH continuous 
irradiation at frequencies MSIP nitric oxide 150.176 - $150.664 \mathrm{GHz}$ for 15 minutes.

Pre continuous 15-minute exposure to EMR THF at frequencies MSIP nitric oxide $150.176-150.664 \mathrm{GHz}$ limits the development of acute and chronic stress reactions by reducing the release of glucocorticoids, including corticosterone by the adrenal cortex. This fact is evidence of anti-stress effect of EMI frequency range. For the first time experimentally justified the use of terahertz electromagnetic waves at frequencies of molecular spectrum of emission and absorption of nitric oxide and atmospheric oxygen to restore the disturbed rheological properties of blood and platelet function. The effect of different modes of electromagnetic radiation at terahertz frequencies MSIP nitric oxide $150.176-150.664 \mathrm{GHz}$ and atmospheric oxygen at $129.0 \mathrm{GHz}$ aggregation activity of platelets, blood rheology in animals in a state of immobilization stress. It is shown that the effects of terahertz waves at frequencies MSIP nitric oxide 150.176 - $150.664 \mathrm{GHz}$ leads to the normalization of disturbed parameters describing platelet aggregation, blood rheology in white rats. The dependence of the efficiency effects of electromagnetic radiation on the specified range of frequencies $150.176-150.664 \mathrm{GHz}$ white rats from the time of exposure to impaired performance of the functional activity of platelets, blood rheology of both males and females with regard to their estrous cycle. The most effective in restoring and preventing the changed parameters of blood rheology in acute stress are continuous and preventive regimens with 15- and 30-minute, and prolonged (chronic) stress 30-minute exposure. Preventive fractional radiation in conditions of acute stress is within 5 minutes limit the development of stress disorders viscosity of blood vessels in the microcirculation.

It is established that the continuous radiation at TGCH impaired by acute stress functional platelet activity of male rats for 30 minutes has a normalizing effect, while the 5- and 15-minute exposure to lead only to a partial recovery of impaired platelet aggregation. Fractional mode TGCH radiation within 5 and 15 minutes restores impaired platelet function caused by acute stress, and more effectively than continuous. Preventive TGCH radiation when exposed for 15 and 30 minutes on male rats is able to prevent the development of characteristic of acute stress reaction violations platelet aggregation. It was revealed that the continuous mode TGCH radiation for 15 and 30 minutes electromagnetic waves at a frequency of molecular spectrum of emission and absorption of atmospheric oxygen $129.0 \mathrm{GHz}$ male rats in a state of acute stress, restores the broken viscous properties of blood, the functional activity of erythrocyte - their aggregation and deformability, as a preventive treatment for the same duration of exposure prevents post stressed violations in terms of blood rheology.

Continuous TGCH radiation within 5 minutes working on white female rats in Dioestrus phases of the estrous cycle and Oestrus in a state of acute stress, electromagnetic waves at a frequency of MSIP atmospheric oxygen 129.0 GHz normalizes the ability of red blood cells in the rat strain in phase Dioestrus 15- and 30-minute exposure fully restore the impaired blood viscosity, and the functional activity of red blood cells regardless of the phase of the estrous cycle. Predictive 5-minute TGCH irradiation of females being Dioestrus barriers development of stress changes the ability of red blood cells to deform, and the 15- and 30-minute exposure completely block post stressed violation of rheological properties of blood regardless of the phase of the estrous cycle.

Continuous exposure to terahertz frequency MSIP atmospheric oxygen $129.0 \mathrm{GHz}$ male rats in acute stress condition for 5 minutes completely restores increased platelet aggregation, 15- and 30-minute modes not only reduced disturbances in platelet function, but also contribute to its further Fractional mode emission reduction by an acute stress sheathe of 15 and 30 minutes is a full recovery of impaired platelet function. Preventive treatment of radiation for 15 and 30 minutes post stressed completely prevents platelet function disorders.

The experimental data provide a basis to extrapolate the use of terahertz radiation at frequencies MSIP nitric oxide $176-150.665,150 \mathrm{GHz}$ and $129.0 \mathrm{GHz}$ atmospheric oxygen in clinical practice to normalize the disturbed parameters of rheological properties of blood and platelet aggregation in patients with different pathologies.

Changes in intravascular component of the microcirculation - qualitative and quantitative composition of red blood cells cause deterioration of the rheological properties of blood during acute and chronic immobilization stress in male rats and female rats that are in one of the two phases of the estrous cycle-Dioestrus or Oestrus. In acute stress immobilization more significantly influence the stress factor for the qualitative and quantitative composition of red blood cells of male rats and female rats under Dioestrus. Female rats in the phase of the estrous cycle of Oestrus were more resistant to the stress factor. Chronic stress violation of quantitative and qualitative composition of red blood cells is similar to those of acute stress.

The absence of the influence of electromagnetic radiation TGCH-band frequencies for MSIP nitric oxide $150.176-150.664 \mathrm{GHz}$ on qualitative and quantitative composition of the red blood cells of intact male rats.

TGCH-radiation at frequencies MSIP nitric oxide $150.176-150.664 \mathrm{GHz}$ partially or completely normalize post stressed violations in the intravascular component of the microcirculation by restoring the quantitative and 
qualitative composition of red blood cells, depending on the mode and the exposure time 15-minute exposure mode is the most effective in restoring impaired qualitative and quantitative composition of red blood cells. All the time modes of preventive TGCH-irradiation of animals in a state of acute stress disorders prevent the development of qualitative and quantitative composition of red blood cells. Daily TGCH pre-irradiation for $30 \mathrm{mi}-$ nutes, the animals in a state of chronic stress only restores the mean corpuscular hemoglobin concentration, and the average diameter and erythrocyte aggregation. The features of the influence of electromagnetic radiation TGCH-band frequencies for MSIP nitric oxide 150.176 - $150.664 \mathrm{GHz}$ on qualitative and quantitative composition of red blood cells white female rats that are in one of the two phases of the estrous cycle-Dyuestrus or Oestrus in a state of acute immobilization exposure. Proactive continuous exposure mode for 15 minutes prevents the development of disturbances in the qualitative and quantitative composition of red blood cells during acute stress. Fractional mode of irradiation for 15 minutes prevents the development of qualitative and quantitative composition of red blood cells during acute stress. Fractional mode of irradiation for 15 minutes against the background of established immobilization stress causes the reduction of the qualitative and quantitative composition of red blood cells in female rats only Oestrus in the phase of the estrous cycle. This mode of irradiation is less effective in normalizing stress disturbed blood rheology in female rats in phase Dioestrus.

Comparative analysis of the effect of continuous and fractional modes TGCH irradiation on the frequency of MSIP oxide nitrogen $150.176-150.664 \mathrm{GHz}$ on the broken qualitative and quantitative composition of the red blood cells of albino rats of both sexes in a state of acute immobilization stress showed that the fractional modes for 5 and 30 minutes more effective than continuous in those time frames. Preliminary fractional 15-minute exposure mode prevents the development of disturbances in the qualitative and quantitative composition of red blood cells.

Effect of pre- and post-irradiation TGCH at frequencies MSIP nitric oxide 150.176 - $150.664 \mathrm{GHz}$ on qualitative and quantitative composition of the red blood cells of albino rats of both sexes with acute immobilization stress is ambiguous. It is shown that pre- and fractional continuous irradiation for 15 animals and preliminary continuous irradiation for 30 minutes post stressed prevents the development of irregularities in the qualitative and quantitative composition of erythrocytes.

First studied the effects of electromagnetic radiation at terahertz frequencies of molecular spectrum of emission and absorption of nitric oxide $150.176-150.664 \mathrm{GHz}$ on morphological and functional microcirculation in various organs of white rats in a state of acute prolonged stress.

It was found that irradiation TGCH capable of restoring the characteristic of acute and long-term immobilization stress microcirculation. This manifests itself in part the normalization of its intravascular component-no signs sladzhsindroma, reducing stasis phenomena: the vascular component- the normalization constant and reducing capillary fragility and extravascular components reducing the severity of edema. It was revealed that the action of the radiation frequency specified in animals in a state of acute immobilization stress observed a significant reduction in ischemic events many internal organs (liver, kidneys, and lungs), and the normalization of blood flow in the brain EMR THF-band frequencies for MSIP nitric oxide $150.176-150.664 \mathrm{GHz}$ causes the downward trend of increased concentrations of endothelin-1 in the blood serum of white male rats in a state of acute immobilization stress. Daily TGCH irradiation for 5 days after each immobilization causes a statistically significant reduction in elevated concentrations of endothelin-1 in the serum of male rats in a state of prolonged stress. Implementation effect TGCH will in the frequencies of molecular spectrum of emission and absorption of nitric oxide $150.176-150.664 \mathrm{GHz}$ on the concentration of endothelin 1 is carried out with the participation of nitric oxide blockade of stress-limiting system of nitric oxide contributes to the development of endothelin 1 .

Normalizing effects of terahertz electromagnetic radiation at frequencies of molecular spectrum of emission and absorption of nitric oxide $150.176-150.664 \mathrm{GHz}$ on microcirculation in animals in a state of acute and long-term immobilization stress realized by changing the activity of the pituitary adrenal and thyroid glands. TGCH-effects at these frequencies moderately reduces hyperactivity gipofizarnonadpochechnikov and thyroid axis stress response, manifested a lack or decrease in expression characteristic of the acute and long-term stress reaction of morphological changes in the pituitary gland, adrenal glands and the thyroid gland.

First studied the effects of electromagnetic radiation at terahertz frequencies MSIP nitric oxide 150.176 $150.664 \mathrm{GHz}$ impaired under stress hemodynamics in the great vessels.

Found that the electromagnetic radiation at these frequencies does not affect the linear flow velocity of intact male albino rats.

The dependence of the efficiency effects of electromagnetic radiation in the terahertz frequency range of mo- 
lecular absorption and emission of nitrogen oxide, $150.176-150.664 \mathrm{GHz}$ in the breakdown of the linear velocity of blood flow in white male rats in a state of acute immobilization stress on the mode of exposure. Thus, with continuous exposure of animals in a state of acute immobilization stress 5-, 15-, and 30-min exposure have the same efficiency as increasing time of exposure does not lead to an increase in efficiency, that is, the most effective is a 5 -minute regime.

Continuous 30-minute regime TGCH-irradiation of animals in a state of acute immobilization stress more effectively restore the basic hemodynamic parameters compared with the fractional regime $\mathrm{TGCH}$ irradiation of the same exposure.

The first time the effectiveness of continuous preventive effect on the frequency range of the electromagnetic spectrum of molecular nitrogen oxide 150.176 - $150.664 \mathrm{GHz}$ stressful violation of linear velocity of blood flow in male rats.

Found that prior immobilization of TGCH irradiation can prevent the development of stress disorders hemodynamics with 5- and 15-minute regimes have equally pronounced effect on the breakdown of the linear velocity of blood flow in the abdominal aorta and femoral artery compared with the same time of continuous exposure modes on the background of the developing acute stress.

The first time the role of endogenous nitric oxide and endothelial NO-synthase involved in the mechanisms of positive coirrecting effects of terahertz radiation at frequencies MSIP nitric oxide $150.176-150.664 \mathrm{GHz}$ performance linear velocity of blood flow in the great vessels of white rats in a state of acute immobilization stress, as evidenced by the lack of normalizing effect of terahertz radiation on disturbed hemodynamics when administered blocker endotolialnoy $\mathrm{NO}$ synthase L-name white male rats in a state of acute stress.

Under the influence of the course of radiation at frequencies of terahertz waves MSIP nitric oxide 150.176 $150.664 \mathrm{GHz}$ on a background of prolonged stress in male rats, a partial normalization of blood flow in the major arteries. Reduced blood flow velocity indicates a decrease in vascular tone under the influence of terahertz waves. The decrease in vascular tone is probably due to the increased production of vasoconstrictor substances by endothelial cells. This is confirmed by the results of the study changes in the concentration of stable nitric oxide metabolites - nitrites and endothelin 1-white rats with prolonged immobilization stress.

The data presented indicate that exposure Courses of terahertz waves at a frequency of 150 MSIP nitric oxide $176-150.664 \mathrm{GHz}$ capable of restoring the impaired blood flow in the arteries of the white rats with prolonged stress. This makes possible the use of irradiation of these waves to restore hemodynamic disturbances occur in pathological conditions.

Studies show that male rats in a state of acute stress and subjected TGCH irradiation at frequencies of molecular spectrum of emission and absorption of nitric oxide $150.176-150.664 \mathrm{GHz}$, there is a restoration of perfusion index (M), while it is not statistically different from the level of the control group. The animals in this group there is a statistically significant compared to the group of animals in a state of acute stress and not subjected TGCH-irradiation, an increase in FLACSO and the coefficient of variation. It should be noted that there is a tendency to increase the values of FLACSO, and especially the coefficient of variation in the animals in this group compared with the control group, indicating a more intense modulation of the microcirculation and mechanisms of its regulation.

These amplitude-frequency analysis of LDF-grams shows TGCH that after irradiation of male rats in a state of acute immobilization stress is statistically significant increase in the amplitude and endothelial vasomotor fluctuations compared to the group of animals in a state of acute immobilization stress is not affected by TGCHeffects. This is reflected in the increase of endothelial vasodilator activity (activation of basal nitric oxide production) and a decrease in peripheral resistance. Also, there is an increase of pulse amplitude (cardiac) vibrations, indicating an increase in the inflow of arterial blood microcircular channel. All figures are amplitude - frequency analysis of LDF - gram animals in this group did not differ significantly from those of the control group.

Thus, the electromagnetic radiation in the terahertz frequency range of molecular absorption and emission of nitrogen oxide increases 150.176 - 150.664 reduced both basal and stimulated production of nitric oxide by the endothelium in male rats in a state of acute immobilization stress. This is consistent with the results of earlier biochemical studies that showed an increased concentration of nitrite levels in male rats in a state of stress immoblizative influenced electromagnetic waves specified frequency range, indicating the activation of nitroxidergic system. Increasing the role of passive mechanisms of regulation of microcirculation (amplitude increase heart rate, reflecting the increase in the inflow of arterial blood into the bloodstream, and to a lesser extent, the 
amplitude of the respiratory rhythm) can be regarded as a compensatory response of tissue reperfusion after ischemia. Increasing the role of active and passive modulation mechanisms microcirculation under the influence of electromagnetic radiation at frequencies MSIP nitric oxide accounts for the inflow of arterial blood microcircular channel, increasing the number of functional capillaries and normalization of perfusion.

Studies indicate that male rats in a state of prolonged stress and subjected to a course of radiation at frequencies of terahertz waves MSIP nitric oxide $150.176-150.664 \mathrm{GHz}$ is a restoration of perfusion index (M), while it is not statistically significantly different from the level of the control group. The animals in this group showed a statistically significant compared to the group of animals in a state of prolonged stress and irradiated terahertz waves, the increase in the standard deviation and the coefficient of variation of perfusion. It should be noted that the value of the standard deviation and coefficient of variation perfusion reaches the level values of the control group, indicating incomplete recovery flow modulation and the mechanisms of its regulation.

These amplitude and frequency analysis of LDF grams show that male rats subjected to irradiation with electromagnetic waves rate at frequencies MSIP nitric oxide in extended immmoblizative stress occurs statistically significant increase in amplitude and endothelial vasomotor oscillations compared to a group of animals in a state of prolonged immobilization stress, do not suffer TGCH-effects. This represents an increase vozodilative activity of endothelium (basal activation of nitric oxide production) and a decrease in peripheral resistance. Also, there is an increase of respiratory and pulse amplitudes (cardiac) oscillations, which indicates an increase in blood flow in the microcirculation. However, all indicators amplitudnochastotnogo analysis of LDF-gram animals in this group was significantly lower than the control group. This indicates a partial restoration of basal nitric oxide production, peripheral resistance and blood flow in the microcirculation.

When analyzing the results of termoprobe of skin found that under the action of waves TGCH male rats in a state of prolonged immobilization stress completely normalized initial and maximum perfusion and perfusion after initial restoration of blood flow. Scaling maximum perfusion in these animals suggests restoring secretion of nitric oxide induced endothelial capillary reserve power animals of this group is significant variability, which covers substantially quartile range as the control group and the group of animals in a state of prolonged stress is not exposed terahertz waves. This indicates that the male rats in a state of prolonged immobilization stress subjected to irradiation rate terahertz waves is partially retained strain generating microvessels, that is initially operates minimal number of capillaries than the animals of the control group.

Continuous and fractional modes of the electromagnetic radiation at a frequency of MSIP $129.0 \mathrm{GHz}$ atmospheric oxygen contributes to the restoration of impaired aggregation activity of male rats in a state of acute stress immoblization. The degree of recovery of impaired platelet function depends on the mode of exposure.

TGCH-efficiency continuous impact on male rats with acute immobilization stress on frequency $129.0 \mathrm{GHz}$ atmospheric oxygen depends on the exposure time. The most effective in restoring impaired platelet aggregation activity of the males is continuous irradiation for 5 minutes, 15- and 30-minute irradiation, however, inhibit platelet aggregation.

The effectiveness of the fractional mode TGCH-effects on male rats with acute immobilization stress on the frequency $129.0 \mathrm{GHz}$ atmospheric oxygen depends on the exposure. The most effective in restoring the impaired platelet aggregation males is fractional mode total exposure of 5 minutes, which partially restores the impaired platelet aggregation, and fractional exposure mode total duration of 30 minutes causes the inhibition of platelet aggregation process.

Comparative analysis of the effect of electromagnetic radiation in the terahertz frequency MSIP atmospheric oxygen $129.0 \mathrm{GHz}$ for the impaired functional activity of platelets in male rats in a state of acute immobilization stress showed that the continuous mode TGCH-efficient fractional irradiation.

For continuous mode of irradiation for 5 minutes more effectively recovers main indicators of platelet aggregation than the fractional mode with the same exposure.

Continuous TGCH impact on the frequency of atmospheric oxygen at $129.0 \mathrm{GHz}$ females Dioestrus phase of the estrous cycle by an acute stress immoblization for 5 minutes only partially contributes to the restoration of impaired platelet aggregation, irradiation for 15 minutes, completely restores the violation of platelet aggregation, 30-minute exposure inhibits the functional activity of platelets.

Comparative analysis of sex differences in reactions to terahertz radiation at a frequency of $129.0 \mathrm{GHz}$ atmosphere oxygen by an acute immobilization stress showed that male albino rats are more sensitive than females in the phase of the estrous cycle to Dioestrus terahertz radiation at a frequency of MSIP atmospheric oxygen $129.0 \mathrm{GHz}$. 
The previous acute immobilization stress TGCH-irradiation on the frequency of atmospheric oxygen MSIP 129.0 $\mathrm{GHz}$ is capable of preventing the development of stress disorders platelet function in male rats. A more pronounced effect has 15- and 30-minute mode, while in continuous mode is 5 minutes of exposure.

The irradiation of white male rats in a state of stress immoblizative TGCH-waves at frequencies MSIP NO resulted in a statistically significant increase of plasma nitrite concentration of male rats in a state of acute stress immoblizative not subjected TGCH-effects. Stable and low concentrations of nitrite in the blood plasma of male rats in a state of acute immobilization stress on the background of L-Name indicates that the realization of the effect TGCH-wave frequency is specified with the participation of NO-synthase components of the cycle NO.

Increased activity of NO, leading to increased concentrations of nitrite in plasma under the influence of terahertz electromagnetic radiation makes it possible to develop new and effective drug-free correction techniques to reduce the endogenous synthesis of NO, which can be used in the treatment of diseases of the cardiovascular system involving production deficit NO (coronary artery disease, hypertension, etc.) studies suggest that exposure to electromagnetic waves in the terahertz frequency range of molecular absorption and emission of nitrogen oxide 150.176 - $150.664 \mathrm{GHz}$ male rats before immobilization can prevent specific to acute immobilization stress perfusion abnormalities microcircular channel skin. Terahertz waves specified frequency effectively prevents the deterioration of the average perfusion and microcirculation disturbance modulation mechanisms. In a comparative analysis of the two exposure modes terahertz waves detected that the average efficiency of effects on microvascular perfusion prior to immobilization when the application TGCH irradiation at frequencies of nitric oxide and terahertz waves impact against developing the same immobilization stress. It was found that animals with prolonged immobilization stress are a persistent decrease in perfusion and blood flow in the modulation of inhibition microcirculation of the skin. These changes are more pronounced in comparison with acute immobilization stress. It is shown that a course of radiation at frequencies of terahertz waves MSIP NO: 150.176 $150.664 \mathrm{GHz}$ after each immobilization of male rats by prolonged stress can partially restore perfusion violation of the circular bed of the skin. This manifests the full normalization of the mean perfusion (perfusion index). Course terahertz wave irradiation of said frequency modulation is only partially normalizes blood flow in the skin microcircular vein of male rats during prolonged immobilization stress, as evidenced by partial restoration of perfusion standard deviation and coefficient of variation.

Terahertz radiation at frequencies MSIP 150.176 - $150.664 \mathrm{GHz}$ partially or completely inhibits the intensity of lipoperosidation reduces the phenomenon autointaksication and cytolysis reactivates all links in the antioxidant and anti-radical protection, depending on the experimental conditions and time of exposure.

The irradiation of animals with acute immobilization stress the most effective was the 30-minute exposure mode. In this case, there was no statistically significant difference from the control group of the studied parameters characterizing the reaction of lipid peroxidation and antioxidant activity of blood. Effect of 5- and 15-minute frequencies of terahertz radiation on the MSIP nitric oxide $150.176-150.664 \mathrm{GHz}$ by an acute stress did not cause immoblizative positive dynamics in terms of the antioxidant system and lipid peroxidation. So, at the 15 minute exposure mode terahertz waves was no change in all of the studied parameters in comparison with the group of animals in a state of acute stress. At the 15-minute exposure occurred only a partial restoration of parameters describing the processes of lipid peroxidation and antioxidant capacity of the blood of animals.

The most effective mode of terahertz radiation of animals on the background of chronic stress was also a 30 minute regime daily for 5 days of exposure. Against the background of this mode of action is completely normalized the activity of the antioxidant system of blood and there was a decrease to the level of intact animals, concentrations of toxic intermediate products of lipid peroxidation. At the 5-minute exposure terahertz waves at frequencies of nitric oxide 150.176 - $150.664 \mathrm{GHz}$ not recovering indicators of antioxidant system and lipid peroxidation, as evidenced by the lack of a statistically significant difference compared with the group of stressed animals. At the 15-minute daily regime for 5 days of exposure of animals by chronic stress is a partial recovery of the parameters studied the antioxidant system and a partial normalization of intermediate concentrations of free radical oxidation products.

Based on the study it can be concluded about the positive impact of terahertz radiation at frequencies of $150.176-150.664 \mathrm{GHz}$ on the changed parameters of the metabolic status of the experimental animals are in a state of acute stress immobilizption. The most effective in normalizing the study parameters is a 30 -minute exposure mode. At the 15-minute exposure mode, there is a positive effect on the indicators of metabolic processes and metabolism (normal triglyceride concentration, the activity of AST and glutathione-S-transferase), and at 5minute mode is absent. 
In conditions of prolonged immobilization shown that in parallel with the action of the stress agent daily for 5 days of irradiation of terahertz waves at frequencies of molecular spectrum of emission and absorption of nitric oxide $150.176-150.664 \mathrm{GHz}$ prevents the development of stress-dependent changes in the metabolic status of male rats which indicates the theoretical possibility of correction of these changes in this type of exposure. Thus, the daily for 5 days, the application of terahertz radiation at frequencies of nitric oxide $150.176-150.664 \mathrm{GHz}$ to 30 minutes there is complete recovery of all the studied parameters that characterize the metabolic activity of the body's internal environment of experimental animals. The daily for 5 days exposure range of said electromagnetic waves for 15 minutes against prolonged stress is observed only a partial normalization of the metabolic status (statistically significantly reduced triglyceride levels). The impact of terahertz radiation at frequencies of nitric oxide $150.176-150.664 \mathrm{GHz}$ to 5 minutes for 5 days in the stressed animals did not cause statistically significant changes in the metabolic status of the studied parameters. On the basis of experimental studies of the "Central Institute of scientific and research instrumentation" (Saratov g) was established EHF therapy "Orbit" in three versions YaKU L 941526001941526 001-01 Yakulov And Yakulov 941526 001-02, for which the Federal Service on surveillance in Healthcare and Social Development issued a license number 99-03-002043 on June 7, 2010 and recorded license NQ FSR 2009/05497 on the production, sale and use of the territory of the Russian Federation These devices have been clinically tested in clinics, Saratov State Medical University, the Moscow Scientific Research Institute of Oncology PA Herzen Moscow city hospital number 15. Published guidelines "Using terahertz therapy in clinical practice" (Saratov, 2011). According to the results of experimental studies, received 24 patents for invention, and 2- the utility model, defended his doctoral dissertation (E. V. Andronov, 2008; O. N. Antipov, 2011), ten PhD theses (M. Bullock, 2001; L. I. Malinova, 2002; N. V. Mamontova, 2006; O. I. Pomoshnikova, 2006; A. N. Ivanov, 2007; S. V. Suhova, 2009; T. S. Velykanova, 2011; T. S. Kiriyazi, 2011; S. V. Svistunov, 2011) prepared for the protection of two doctoral dissertations (A. N. Ivanov; A. A. Tsymbal).

\section{References}

[1] Golant, M.B., Bryukhova, A.K., Dvadtsatova, E.A., et al. (1983) The Possibility of Regulating the Activity of Microorganisms When Exposed to Electromagnetic Waves MM Range. In: Devyatkova, N.D., Ed., The Effects of Non-Sexual Effects of Millimeter-Wave Radiation on Biological Objects, M. IRE RAS, 115-122.

[2] Devyatkov, N.D. and Betsky, O.V. (1985) Peculiarities of Interaction of Low-Intensity Millimeter Radiation with Biological Objects - The Use of MM-Radiation of Low Intensity in Biology and Medicine. Scientific Digest, 108-117.

[3] Golant, M.B. (1986) Effect of Low-Intensity Monochromatic Electromagnetic Radiation of Millimeter Range on Biological. Biophysics, 31, 6-11.

[4] Devyatkov, N.D., Golant, M.B. and Betsky, O.V. (1994) Features of Biomedical Applications of Millimeter Wave. M, IRE, RAS, 160.

[5] Kiruchuk, V.F. Golovacheva, T.V. and Chizh, A.G. (1999) EHF-Therap. Saratov State Medical University Publishing House, Saratov, 360.

[6] Kirichuk, V.F., Andronov, E.V., Mayborodin, A.V., et al. (2004) Effect of Electromagnetic Radiation at a Frequency Range of Molecular Absorption and Emission of Nitrogen Oxide $(240 \mathrm{GHz})$ on the Aggregation Activity of Platelets in Patients with Unstable Angina. Angiology and Vascular Surgery, 10, 12. 